\title{
INFLUÊNCIA DO CALCÁRIO E DO SILICATO DE CÁLCIO E MAGNÉSIO COM GESSO AGRÍCOLA NO NÚMERO DE PERFILHOS DE Panicum maximum JACQ. CV. CAPIM MOMBAÇA
}

\author{
Vinícius Faúla Aguiar ${ }^{1}$ \\ Rosália Nazareth Rosa Trindade ${ }^{2}$ \\ Cleicimar Gomes da Costa ${ }^{3}$ \\ Rodolfo Luiz Carvalhais Lima ${ }^{4}$ \\ Kátia Cristina da Silva ${ }^{5}$ \\ Lucas Anderson Fernandes Cordeiro ${ }^{6}$ \\ Alisson José Eufrásio de Carvalho ${ }^{7}$ \\ Aderlan Gomes da Silva ${ }^{8}$
}

Resumo: O objetivo deste trabalho é avaliar o efeito das correções do solo efetuadas com calcário e silicato de cálcio e magnésio no aumento do número de perfilhos das plantas de Panicum maximum Jacq. Cultivar capim Mombaça. Foi adotado delineamento experimental em blocos ao acaso, em esquema fatorial $2 \times 5$, sendo estudado em quatro blocos o efeito de duas fontes de corretivo e cinco métodos de aplicação, além da testemunha. Nas análises onde foi observada significância estatística, realizou-se teste de Tukey a 5,0\% de probabilidade. A unidade experimental é constituída por vasos (colunas) de tubos de PVC de quinze centímetros de diâmetro por sessenta de altura, totalizando 44 unidades. A contagem do número de perfilhos foi realizada no momento dos 04 cortes da forragem. Os perfilhos foram mensurados em cada um dos 04 tubos (repetições) referentes a cada tratamento por planta. 0 tratamento 06 apresentou maior unidade de perfilho por planta.

Palavras-chave: Corretivo; Forragem; Manejo intensivo; Produtividade.

\footnotetext{
1 Agronomia/Instituto Federal de Educação, Ciência e Tecnologia de Minas Gerais, Brasil. E-mail: vinicius.aguiar.agr@gmail.com.

2 Universidade Federal dos Vales do Jequitinhonha e Mucuri/Departamento de Engenharia Florestal, Brasil. Email: rosalia.trindade22@gmail.com.

3 Agronomia/Instituto Federal de Educação, Ciência e Tecnologia de Minas Gerais, Brasil. E-mail: cleicimar_gomesje@hotmail.com.

4 Agronomia/Instituto Federal de Educação, Ciência e Tecnologia de Minas Gerais, Brasil. E-mail: rodolfocarvalhais93@gmail.com.

5 Agronomia/Instituto Federal de Educação, Ciência e Tecnologia de Minas Gerais, Brasil. E-mail: katiaeaf@yahoo.com.br.

6 Agronomia/Instituto Federal de Educação, Ciência e Tecnologia de Minas Gerais, Brasil. E-mail: lucasanderson02@gmail.com.

7 Agronomia/Instituto Federal de Educação, Ciência e Tecnologia de Minas Gerais, Brasil. E-mail: alisson.carvalho@ifmg.edu.br.

8 Agronomia/Instituto Federal de Educação, Ciência e Tecnologia de Minas Gerais, Brasil. E-mail: aderlan.silva@ifmg.edu.br.
} 